ЕЛЕКТРОНІКА

РАДІОТЕХНІКА

ЗАСОБИ ТЕЛЕКОМУНІКАЦІЙ

ELECTRONICS

RADIO ENGINEERING

TELECOMMUNICATION FACILITIES

UDK 621.371+537.8:621.372

I.Yu. Dmitrieva, PhD, Assoc.Prof.

Odessa National Academy of Telecommunications named after O.S. Popov, 1 Koval'ska Str., 65029 Odessa, Ukraine; e-mail: irina.dm@mail.ru

\title{
DETAILED EXPLICIT SOLUTION OF THE ELECTRODYNAMIC WAVE EQUATIONS
}

\begin{abstract}
І.Ю. Дмитрісва. Детальне точне розв'язання електродинамічних хвильових рівнянь. Дані результати стосуються загального наукового напрямку відносно математичного моделювання і аналітичного вивчення явищ електромагнітного поля, що описуються системами диференціальних рівнянь у частинних похідних. Спеціальний електродинамічний інженерний процес з експофункціональними впливами моделюється диференціальною системою Максвелла, чиє ефективне дослідження еквівалентне строгому розв'язанню загального хвильового диференціального рівняння у частинних похідних, яке залежить від усіх скалярних компонент векторів напруженості електромагнітного поля. Це рівняння розв'язується детально у явному вигляді методом інтегральних перетворень та безвідносно конкретних крайових умов. Розглянуто спеціальні випадки незбуджених вакууму й ізотропного однорідного середовища. Запропонований підхід може застосовуватись до довільної кінцево вимірної системи диференціальних рівнянь у частинних похідних з кусково-сталими коефіцієнтами та відповідних скалярних хвильових рівнянь, які є математичними моделями в сучасній електродинаміці. У порівнянні з відомими результатами дане дослідження є повністю детальним та точним, що гарантує його безпосереднє практичне застосування.

Ключеві слова: диференціальна система Максвелла, загальне хвильове рівняння відносно усіх скалярних компонент векторів напруженості електромагнітного поля, детальний точний розв'язок.

I.Yu. Dmitrieva. Detailed explicit solution of the electrodynamic wave equations. Present results concern the general scientific tendency dealing with mathematical modeling and analytical study of electromagnetic field phenomena described by the systems of partial differential equations. Specific electrodynamic engineering process with expofunctional influences is simulated by the differential Maxwell system whose effective research is equivalent to the rigorous solution of the general wave partial differential equation regarding all scalar components of electromagnetic field vector intensities. The given equation is solved explicitly in detail using method of integral transforms and irrespectively to the concrete boundary conditions. Specific cases of unexcited vacuum and isotropic homogeneous medium were considered. Proposed approach can be applied to any finite dimensional system of partial differential equations with piece wise constant coefficients and its corresponding scalar equations representing mathematical models in modern electrodynamics. In comparison with the known results, current research is completely thorough and accurate that implies its direct practical application.

Keywords: differential Maxwell system, general wave equation regarding all scalar components of electromagnetic field vector intensities, detailed explicit solution.
\end{abstract}

Introduction. Since majority of electromagnetic field phenomena is described mathematically by the systems of partial differential equations (PDEs), their exact solution remains always required. 
Literature review. The first step here concerns the so called operator diagonalization method. It reduces the original matrix statement to the equivalent union of scalar problems where each of them depends on the only one component of the unknown vector field function [1]. The second stage of study is the mathematical simulation in terms of the corresponding boundary problems which engineering and analytic correctness is based on the preceding diagonalization procedure [2]. Moreover, essential simplicity of scalar formulation in comparison with the initial matrix structure strongly supports necessity of those aforesaid diagonalization methods.

Nevertheless, if the concrete applied problem is solved explicitly, it rather often happens that obtained formulae are not specified in all desired details. Though for mathematicians such shortcoming is not of great importance, in practical engineering research it can become a serious obstacle. Really, in this case, direct computation appears impossible because final result is not complete. In its turn, the value of analytic approach is lost. Thus in [3], various electrodynamic wave equations were considered. They represented particular cases of the general wave that was equivalent to the differential Maxwell system for the expofunctionally excited media [1]. Though suggested solutions were rigorous, they could not be directly applicable because some important interim expressions were missed. Naturally, relevant boundary problems mathematically modeling engineering electrodynamic processes were not solved. This disadvantage of study causes the aim of the present article.

Aim of the Research consists in detailed complete explicit solution of electrodynamic wave equations in the specific cases of media.

Main Body. The general wave equation regarding all scalar components of the unknown electromagnetic field vector intensities $\vec{E}, \vec{H}$ was obtained in [1]

$$
\tilde{\partial}_{0}^{2}\left(\tilde{\partial}_{0}^{2}-\Delta\right) F_{k i}=\left(\partial_{i}^{2}-\tilde{\partial}_{0}^{2}\right) \varphi_{k i}+\partial_{i}\left(\partial_{v} \varphi_{k v}+\partial_{l} \varphi_{k l}\right) ; v \neq l, i \neq v, i \neq l,(i, v, l=\overline{1,3} ; k=1,2) \text {. }
$$

$\operatorname{In}(1)$

$$
\begin{gathered}
\vec{F}_{1}=\vec{E}, \vec{F}_{2}=\vec{H} ; \quad \vec{\varphi}_{1}=A \vec{e}^{C T}+P \vec{j}^{C T}, \quad \vec{\varphi}_{2}=C \vec{e}^{C T}-A \vec{j} C T ; \\
\vec{F}_{k}=\left\{F_{k i}\right\}_{i=1}^{3}, \quad F_{k i}=F_{k i}(x, y, z, t), \quad(k=1,2) ; \\
\vec{\varphi}_{k}=\left\{\varphi_{k i}\right\}_{i=1}^{3}, \quad \varphi_{k i}=\varphi_{k i}(x, y, z, t), \quad(k=1,2) ; A=\operatorname{rot}, C=\sigma+\varepsilon_{a} \partial_{0}^{*}, \quad P=r+\mu_{a} \partial_{0}^{*}, \quad \partial_{0}^{*}=\partial_{0} \pm \lambda,
\end{gathered}
$$

where $\Delta=\sum_{i=1}^{3} \partial_{i}^{2}$;

$$
\begin{aligned}
& \partial_{0}=\partial / \partial t, \partial_{1}=\partial / \partial x, \quad \partial_{2}=\partial / \partial y, \quad \partial_{3}=\partial / \partial z, \\
& \tilde{\partial}_{0}^{2}=P C=\mu_{a} \varepsilon_{a}\left(\partial_{0}^{*}\right)^{2}+\left(\sigma \mu_{a}+r \varepsilon_{a}\right) \partial_{0}^{*}+r \sigma
\end{aligned}
$$

$\sigma, \mu_{a}, \varepsilon_{a}=$ const $>0$ are the specific conductivity, absolute magnetic and electric permeability of the medium accordingly.

The given functions $\vec{j}^{C T}, \vec{e}^{C T}=\vec{j}^{C T}, \vec{e}^{C T}(x, y, z, t)$ describe the outside current sources and intensities; $r$ is the theoretical positive constant; $\lambda=$ const $>0$ is the signal parameter exciting the medium. Activity of the medium corresponds to "-", and "+" means absorption of signal by medium.

It is clear, that the general wave PDE (1), (2) for the expofunctionally excited media can be rewritten uniformly

$$
\tilde{\partial}_{0}^{2}\left(\tilde{\partial}_{0}^{2}-\Delta\right) F=f
$$

where $F=F(x, y, z, t), f=f(x, y, z, t)$ are the unknown and the given functions from the left and the right parts of (1) accordingly.

Further results concern detailed explicit solution of (3) in various media.

\section{Results.}

Unexcited vacuum. If $\vec{e}^{C T} \equiv 0$, the studied unexcited $(\lambda=0)$ isotropic medium is vacuum $(\sigma=0)$, and $r=0$, the simplest though important specific case of (3) appears 


$$
\partial_{0}^{2}\left(\partial_{0}^{2}-\frac{\Delta}{\mu_{a} \varepsilon_{a}}\right) F=\tilde{f} ; \quad \tilde{f}=\frac{f}{\left(\mu_{a} \varepsilon_{a}\right)^{2}},
$$

where $f$ follows from (1), (2)

$$
\begin{gathered}
f=\left(\partial_{i}^{2}-\mu_{a} \varepsilon_{a} \partial_{0}^{2}\right) \varphi_{k i}+\partial_{i}\left(\partial_{v} \varphi_{k v}+\partial_{l} \varphi_{k l}\right) ; v \neq l, i \neq v, i \neq l,(i, v, l=\overline{1,3} ; k=1,2), \\
\vec{\varphi}_{1}=\mu_{a} \partial_{0} \vec{j}^{C T}, \quad \vec{\varphi}_{2}=-\operatorname{rot} \vec{j}^{C T} .
\end{gathered}
$$

It is easy to notice that in spite of the common differential operator from the left part in (4), the unknown $F$ and the given $f$ scalar functions are different for each case of $k, l, i, v$ from (5). This fact is based on (1), (2).

Explicit solution of (4), (5) can be obtained using the method of integral transforms [4] by all spatial variables $(x, y, z)$ and not touching time argument $t$ that is accepted as the main one. Simplification of future computation is supported by the following table of symbols: spatial variables from $\mathbf{R}_{3}$ are renamed into $x=x_{1}, y=x_{2}, z=x_{3}$; the kernel of the $i$-th integral transform by the argument $x_{i}$ with parameter $p_{i}$ is

$$
K_{i}=K_{i}\left(x_{i}, p_{i}\right)
$$

and direct integral transform is determined by

$$
S_{i}=\int_{a_{i}}^{b_{i}} K_{i}\left(x_{i}, p_{i}\right) d x_{i},
$$

where $a_{i}, b_{i}$ are the initial and the end points of the open contour of integration $L_{i},(i=\overline{1,3})$.

Values of $a_{i}, b_{i}$ can be either finite or infinite real, or complex as well [4]. For analysis of influence of integral transforms upon $\Delta F,(x, y, z)=\left\{x_{i},(i=\overline{1,3})\right\}$, the common scalar component of electromagnetic field intensity is rewritten like

$$
F=F(x, y, z, t)=F\left(x_{i},(i=\overline{1,3}) ; t\right) .
$$

After application of the $i$-th integral transform to $\Delta F=\sum_{i=1}^{3} \partial_{i}^{2} F$ and double integration by parts, the following expression is got

$$
\begin{gathered}
\int_{a_{i}}^{b_{i}}\left(\partial_{i}^{2} F\right) K_{i}\left(x_{i}, p_{i}\right) d x_{i}=\left.\left(K_{i}\left(\partial_{i} F\right)-\left(\partial_{i} K_{i}\right) F\right)\right|_{x_{i}=a_{i}} ^{b_{i}}+\int_{a_{i}}^{b_{i}}\left(\partial_{i}^{2} K_{i}\right) F d x_{i}= \\
=s_{i}\left(p_{i}, x_{v}(v \neq i ; v=\overline{1,3}) ; t\right)+\eta_{i}\left(p_{i}\right)_{i} F_{t r}, \quad(i=\overline{1,3}),
\end{gathered}
$$

where

$$
s_{i}=s_{i}\left(p_{i}, x_{v}(v \neq i ; v=\overline{1,3}) ; t\right)=\left.\left(K_{i}\left(\partial_{i} F\right)-\left(\partial_{i} K_{i}\right) F\right)\right|_{x_{i}=a_{i}} ^{b_{i}}=s_{i}\left(p_{i}, x_{v}, x_{l}, t\right),(v, l \neq i ; l>v),
$$

the right or the left inferior index " $t r$ " everywhere in this paper implies conversion to the respective transform.

Though in (7) $i, v, l=\overline{1,3}$, but because of two last inequalities from (7), $v, l$ possess only two values from three possible. Moreover, the second item $\eta_{i}\left(p_{i}\right)_{i} F_{t r}$ from the right part of (6) has the factor $\eta_{i}\left(p_{i}\right)$ dependent only on the parameter $p_{i}$ of the $i$-th integral transform. This item appears owing to the operation $\partial_{i}^{2} K_{i},(i=\overline{1,3})$.

At last, "incomplete" $i$-th transform of $F$ by variable $x_{i}$ 


$$
{ }_{i} F_{t r}={ }_{i} F_{t r}\left(p_{i}, x_{v}(v \neq i ; v=\overline{1,3}) ; t\right)=\int_{a_{i}}^{b_{i}} F\left(x_{i}(i=\overline{1,3}) ; t\right) K_{i}\left(x_{i}, p_{i}\right) d x_{i}=\int_{a_{i}}^{b_{i}} F K_{i} d x_{i}, \quad(i=\overline{1,3}) .
$$

Basing on (8), the "complete" transform of $F$ by all spatial variables $(x, y, z)=\left(x_{i}, i=\overline{1,3}\right)$ is

$$
\begin{aligned}
F_{t r}=F_{t r}\left(p_{i}(i=\overline{1,3}) ; t\right)=F_{t r}(p, t) & =\left(\prod_{\substack{v=1 \\
v \neq i}}^{3} \int_{a_{v}}^{b_{v}} K_{v} d x_{v}\right){ }_{i} F_{t r}=\int_{a_{l}}^{b_{l}} \int_{a_{v}}^{b_{v}} K_{v}\left(x_{v}, p_{v}\right) K_{l}\left(x_{l}, p_{l}\right)_{i} F_{t r}\left(p_{i}, x_{v}, x_{l}, t\right) d x_{v} d x_{l} ; \\
p & =\bigcup_{i=1}^{3} p_{i}=\left(p_{1}, p_{2}, p_{3}\right), \quad(i=\overline{1,3}),
\end{aligned}
$$

where conditions for $v, l$ remain the same as for $s_{i},(i=\overline{1,3})$ in (7).

Application of those required integral transforms by all spatial variables $(x, y, z)=\left(x_{i}, i=\overline{1,3}\right)$ satisfying the specific engineering problem statement, takes (4), (5) into ordinary differential equation (ODE) regarding corresponding transforms dependent on time argument $t$

$$
\frac{d^{2}}{d t^{2}}\left(\frac{d^{2}}{d t^{2}}-\frac{\Delta_{t r}}{\mu_{a} \varepsilon_{a}}\right) F_{t r}=f_{t r}^{*}
$$

$\operatorname{In}(10)$

$$
\Delta_{t r}=\Delta_{t r}(p)=\sum_{i=1}^{3} \eta_{i}\left(p_{i}\right) ; \quad f_{t r}^{*}=\tilde{f}_{t r}-\sum_{i=1}^{3}\left(\prod_{\substack{v=1 \\ v \neq i}}^{3} \int_{a_{v}}^{b_{v}} K_{v} d x_{v}\right) S_{i}, \quad(i=\overline{1,3}) ; \quad \tilde{f}_{t r}=\frac{f_{t r}}{\left(\mu_{a} \varepsilon_{a}\right)^{2}},
$$

and the product under the sum symbol $\Sigma$ at the second expression from (11) looks similarly to (9):

$$
\left(\prod_{\substack{v=1 \\ v \neq i}}^{3} \int_{a_{v}}^{b_{v}} K_{v} d x_{v}\right) s_{i}=\int_{a_{l}}^{b_{l} b_{v}} \int_{a_{v}} K_{v}\left(x_{v}, p_{v}\right) K_{l}\left(x_{l}, p_{l}\right) s_{i}\left(p_{i}, x_{v}, x_{l}, t\right) d x_{v} d x_{l}, \quad(v, l \neq i ; l>v ; i, v, l=\overline{1,3}) .
$$

Additionally, in (11) $f_{t r}$ represents "complete" transform for $f$ from (5) and is defined by its all "incomplete" transforms, as it is shown in (9).

Since the aim of the research consists in the really rigorous solution of (10), (11) generated by the originally formulated problem (4), (5), the explicit expression of $f_{t r}$ should be proposed in detail. The external differential operator in (5) is $\partial_{i}$. So it seems more natural to apply to $f$ integral transform by $x_{i}$. Integration by parts in (4) with support of (6)...(8), gives "incomplete" $i$-th transform for $f$

$$
\begin{gathered}
{ }_{i} f_{t r}=q_{i}+\left(\eta_{i}\left(p_{i}\right)-\mu_{a} \varepsilon_{a} \partial_{0}^{2}\right)_{t r} \varphi_{k i, i}+\left.\left(\partial_{v} \varphi_{k v}+\partial_{l} \varphi_{k l}\right) K_{i}\right|_{x_{i}=a_{i}} ^{b_{i}}-\int_{a_{i}}^{b_{i}}\left(\partial_{i} K_{i}\right)\left(\partial_{v} \varphi_{k v}+\partial_{l} \varphi_{k l}\right) d x_{i}, \\
v \neq l, i \neq v, i \neq l,(i, v, l=\overline{1,3} ; k=1,2) .
\end{gathered}
$$

The $i$-th "incomplete" transform of $\varphi_{k i}$ from (12) is

$$
\begin{gathered}
{ }_{t r} \varphi_{k i, i}={ }_{t r} \varphi_{k i, i}\left(p_{i}, x_{v}(v \neq i ; v=\overline{1,3}) ; t\right)=\int_{a_{i}}^{b_{i}} \varphi_{k i}\left(x_{i}(i=\overline{1,3}) ; t\right) K_{i}\left(x_{i}, p_{i}\right) d x_{i}=\int_{a_{i}}^{b_{i}} \varphi_{k i} K_{i} d x_{i}, \\
q_{i}=q_{i}\left(p_{i}, x_{v}(v \neq i ; v=\overline{1,3}) ; t\right)=\left.\left(K_{i}\left(\partial_{i} \varphi_{k i}\right)-\left(\partial_{i} K_{i}\right) \varphi_{k i}\right)\right|_{x_{i}=a_{i}} ^{b_{i}}=q_{i}\left(p_{i}, x_{v}, x_{l}, t\right),(v, l \neq i ; l>v),
\end{gathered}
$$


and factor $\eta_{i}\left(p_{i}\right)$ remains the same as earlier in (6). It is explained by the analogous integration by parts, as it is done in (6). Besides, all integral transformations are common for the whole (4). Therefore, when the first two items are obtained in (12), operation $\partial_{i}^{2} K_{i}$ gives the result equivalent to (6). Basing on (12)...(14), the "complete" detailed transform of $f$ is

$$
\begin{gathered}
f_{t r}=f_{t r}(p, t)=\int_{a_{v}}^{b_{v}} \int_{a_{l}}^{b_{l}} K_{v} K_{l}\left(q_{i}+\left(\partial_{v} \varphi_{k v}+\partial_{l} \varphi_{k l}\right) K_{i} l_{x_{i}=a_{i}}^{b_{i}}\right) d x_{l} d x_{v}+\left(\eta_{i}\left(p_{i}\right)-\mu_{a} \varepsilon_{a} \frac{d^{2}}{d t^{2}}\right){ }_{t r} \varphi_{k i}- \\
\int_{a_{i}}^{b_{i}}\left(\partial_{i} K_{i}\right)\left(\int_{a_{l}}^{b_{l}} K_{l}\left(\left.\varphi_{k v} K_{v}\right|_{x_{v}=a_{v}} ^{b_{v}}-\int_{a_{v}}^{b_{v}} \varphi_{k v}\left(\partial_{v} K_{v}\right) d x_{v}\right) d x_{l}+\int_{a_{v}}^{b_{v}} K_{v}\left(\left.\varphi_{k l} K_{l}\right|_{x_{l}=a_{l}} ^{b_{l}}-\int_{a_{l}}^{b_{l}} \varphi_{k l}\left(\partial_{l} K_{l}\right) d x_{l}\right) d x_{v}\right) d x_{i},
\end{gathered}
$$

where ${ }_{t r} \varphi_{k i}(i=\overline{1,3} ; k=1,2)$ is the "complete" transform of $\varphi_{k i}(i=\overline{1,3} ; k=1,2)$; integrands inside of the round brackets are got by the respective integration by parts.

Orders of integration are rearranged in (15) owing to the commutativity in pairs of the partial differential operators.

Further substitution

$$
\frac{d^{2} F_{t r}}{d t^{2}}=\Phi=\Phi(t, p)
$$

reduces (10) to the equivalent simplified wave ODE in terms of transforms of electromagnetic intensities

$$
\left(\frac{d^{2}}{d t^{2}}-\frac{\Delta_{t r}}{\mu_{a} \varepsilon_{a}}\right) \Phi_{t r}=f_{t r}^{*}
$$

Characteristic (performance) equation [5] of (17) has two simple roots

$$
\gamma_{1,2}= \pm \sqrt{\frac{\Delta_{t r}}{\mu_{a} \varepsilon_{a}}} .
$$

Since $\mu_{a}, \varepsilon_{a}=$ const $>0$ according to the original problem statement, the numerical values of (18) are completely determined by the sign of $\Delta_{t r}$. The latter leads to three possibilities of existence for roots (18):

$$
\begin{aligned}
& -\Delta_{t r}>0 ; \\
& -\Delta_{t r}<0 ; \\
& -\Delta_{t r} \in \mathbf{C} .
\end{aligned}
$$

In its turn, the last three conditions evoke three different fundamental systems of solutions (FSS) [5] for the homogeneous equation respective to (17). Later, inhomogeneous ODE (17) is solved uniformly in all three cases using method of arbitrary constant variation [5]. Thus, let at first $\Delta_{t r}>0$. Then FSS is the following $\left\{e^{\gamma \mathrm{t}}, e^{-\gamma \mathrm{t}}\right\}$, with $\gamma=\sqrt{\frac{\Delta_{t r}}{\mu_{a} \varepsilon_{a}}}$, and creates the general solution of (17)

$$
\Phi=C_{1}(t, p) e^{\gamma t}+C_{2}(t, p) e^{-\gamma t} .
$$

The unknown functions $C_{j}(t)=C_{j}(t, p),(j=1,2)$ in (19) are sought from the system [5]

$$
\left\{\begin{array}{l}
C_{1}^{\prime}(t) e^{\gamma t}+C_{2}^{\prime}(t) e^{-\gamma t}=0 \\
\gamma C_{1}^{\prime}(t) e^{\gamma t}-\gamma C_{2}^{\prime}(t) e^{-\gamma t}=f_{t r}^{*}
\end{array}\right.
$$

and are described by the formulae

$$
C_{1}(t)=\frac{1}{2 \gamma} \int e^{-\gamma t} f_{t r}^{*} d t+C_{1}^{*}(p), \quad C_{2}(t)=-\frac{1}{2 \gamma} \int e^{\gamma t} f_{t r}^{*} d t+C_{2}^{*}(p) .
$$


Taking into account (16) and appropriate specific transformed boundary conditions for initial wave equation (4), the unknowns $C_{j}^{*}(p),(j=1,2)$ in (20) are found explicitly. Substituting (20) for (19), one gets exact expression of the unknown general solution for (17)

$$
\Phi(t, p)=\frac{1}{2 \gamma}\left(e^{\gamma t}\left(\int e^{-\gamma t} f_{t r}^{*} d t+C_{1}^{*}(p)\right)-e^{-\gamma t}\left(\int e^{\gamma t} f_{t r}^{*} d t+C_{2}^{*}(p)\right)\right) .
$$

Turning to (16), applying inverse integral operators regarding those direct ones from the left part of (16) and then using (21), the final constructive solution of (10) is got while the first condition takes place

$$
F_{t r}(t, p)=\iint \Phi(t, p) d t^{2}=\frac{1}{2 \gamma} \iint\left(e^{\gamma t}\left(\int e^{-\gamma t} f_{t r}^{*} d t+C_{1}^{*}(p)\right)-e^{-\gamma t}\left(\int e^{\gamma t} f_{t r}^{*} d t+C_{2}^{*}(p)\right)\right) d t^{2} .
$$

Let now the second version $\Delta_{t r}<0$ be considered. The roots (18) become $\pm i \sqrt{\frac{-\Delta_{t r}}{\mu_{a} \varepsilon_{a}}} \in \mathbf{C}$, fraction under the radical symbol is positive and $i=\sqrt{-1}$. Using notation

$$
\gamma=\sqrt{\frac{-\Delta_{t r}}{\mu_{a} \varepsilon_{a}}}>0
$$

the FSS for the homogeneous equation regarding (17) is written $-\{\cos \gamma t \pm i \sin \gamma t\}$, and it forms the general solution of (17)

$$
\Phi=C_{1}(t, p)(\cos \gamma t+i \sin \gamma t)+C_{2}(t, p)(\cos \gamma t-i \sin \gamma t) .
$$

In (24), the unknown functions $C_{j}(t)=C_{j}(t, p),(j=1,2)$ are found as the solutions of system [5]

$$
\left\{\begin{array}{l}
C_{1}^{\prime}(\cos \gamma t+i \sin \gamma t)+C_{2}^{\prime}(\cos \gamma t-i \sin \gamma t)=0 ; \\
C_{1}^{\prime}(-\gamma \sin \gamma t+i \gamma \cos \gamma t)+C_{2}^{\prime}(-\gamma \sin \gamma t-i \gamma \cos \gamma t)=f_{t r}^{*},
\end{array} C_{j}^{\prime}=\frac{\partial C_{j}}{\partial t},(j=1,2) .\right.
$$

Substituting solution of (25)

$$
C_{1,2}(t)=\mp \frac{1}{2 \gamma} \int( \pm \sin \gamma t+i \cos \gamma t) f_{t r}^{*}(t, p) d t+C_{1,2}^{*}(p)
$$

for (24), after simple computation one gets

$$
\Phi=\frac{1}{\gamma}\left(\sin (\gamma t) \int(\cos \gamma t) f_{t r}^{*}(t, p) d t-\cos (\gamma t) \int(\sin \gamma t) f_{t r}^{*}(t, p) d t+\left(C_{2}^{*}-C_{1}^{*}\right) e^{-i \gamma t}\right),
$$

where $C_{j}^{*}(p),(j=1,2)$ have the same meaning as in (20) ... (22).

Similarly to (22), application of the double integral operation to (16) in the presence of (26), leads to the general solution of (10) in the second case

$$
\begin{gathered}
F_{t r}(t, p)=\iint \Phi(t, p) d t^{2}= \\
=\frac{1}{\gamma} \iint\left(\sin (\gamma t) \int \cos (\gamma t) f_{t r}^{*}(t, p) d t-\cos (\gamma t) \int \sin (\gamma t) f_{t r}^{*}(t, p) d t+\left(C_{2}^{*}-C_{1}^{*}\right) e^{-i \gamma t}\right) d t^{2}
\end{gathered}
$$

The last third version $\Delta_{t r} \in \mathbf{C}$, mathematically implies

$$
\begin{gathered}
\frac{\Delta_{t r}}{\mu_{a} \varepsilon_{a}}=\alpha+i \beta \in \mathbf{C} ; \\
\alpha=\operatorname{Re} \frac{\Delta_{t r}}{\mu_{a} \varepsilon_{a}}, \beta=\operatorname{Im} \frac{\Delta_{t r}}{\mu_{a} \varepsilon_{a}} \in \mathbf{R},
\end{gathered}
$$


where

$$
\gamma=\sqrt{\frac{\Delta_{t r}}{\mu_{a} \varepsilon_{a}}}= \pm \frac{1}{\sqrt{2}}\left(\sqrt{\alpha+\sqrt{\alpha^{2}+\beta^{2}}}+i \frac{\beta}{\sqrt{\alpha+\sqrt{\alpha^{2}+\beta^{2}}}}\right), \quad \gamma_{1}=\operatorname{Re} \gamma, \gamma_{2}=\operatorname{Im} \gamma
$$

is easily found.

Then the desired general solution of (17) is

$$
\Phi=C_{1}(t, p) \exp \left(\gamma_{1} t\right)\left(\cos \gamma_{2} t+i \sin \gamma_{2} t\right)+C_{2}(t, p) \exp \left(-\gamma_{1} t\right)\left(\cos \gamma_{2} t-i \sin \gamma_{2} t\right),
$$

and the unknown functions $C_{j}(t)=C_{j}(t, p),(j=1,2)$ represent the solutions of the following system [5]

$$
\left\{\begin{array}{l}
C_{1}^{\prime} \exp \left(\gamma_{1} t\right)\left(\cos \gamma_{2} t+i \sin \gamma_{2} t\right)+C_{2}^{\prime} \exp \left(-\gamma_{1} t\right)\left(\cos \gamma_{2} t-i \sin \gamma_{2} t\right)=0 ; \\
\left(\gamma_{1}+i \gamma_{2}\right)\left(C_{1}^{\prime} \exp \left(\gamma_{1} t\right)\left(\cos \gamma_{2} t+i \sin \gamma_{2} t\right)+C_{2}^{\prime} \exp \left(-\gamma_{1} t\right)\left(-\cos \gamma_{2} t+i \sin \gamma_{2} t\right)\right)=f_{t r}^{*},
\end{array} C_{j}^{\prime}=\frac{\partial C_{j}}{\partial t}\right.
$$

and

$$
C_{1,2}(t)= \pm \frac{1}{2\left(\gamma_{1}+i \gamma_{2}\right)} \int \frac{\exp \left(\mp \gamma_{1} t\right) f_{t r}^{*}(t, p) d t}{\cos \gamma_{2} t \pm i \sin \gamma_{2} t}+C_{1,2}^{*}(p)
$$

In (30), $C_{j}^{*}(p),(j=1,2)$ keep the same information as earlier for the preceding first and second versions. Substitution of (30) into (29) gives the required general solution of (17)

$$
\Phi=\frac{1}{2 \gamma}\left(e^{\gamma t} \int e^{-\gamma t} f_{t r}^{*}(t, p) d t-\mathrm{e}^{-\gamma t} \int \mathrm{e}^{\gamma t} f_{t r}^{*}(t, p) d t+C_{1}^{*} e^{\gamma t}-C_{2}^{*} e^{-\gamma t}\right),
$$

where $\gamma$ is from (28).

At last, returning to (16), using double integration operation in (16), the general solution of the transformed wave equation (10) for the third version is

$$
F_{t r}(t, p)=\iint \Phi(t, p) d t^{2}=\frac{1}{2 \gamma} \iint\left(e^{\gamma t}\left(\int e^{-\gamma t} f_{t r}^{*} \mathrm{~d} t\right)-e^{-\gamma t}\left(\int e^{\gamma t} f_{t r}^{*} d t\right)+C_{1}^{*} e^{\gamma t}-C_{2}^{*} e^{-\gamma t}\right) d t^{2},
$$

where $\gamma$ is identical to (31), (28);

$\Phi$ is chosen from (31).

Comparing (32), (22), it is easy to find that in both first and third cases, while $\gamma \in \mathbf{R}$ and $\gamma \in \mathbf{C}$, the unknown general solution for (10) can be written uniformly

$$
F_{t r}=F_{t r}(t, p)=\frac{1}{2 \gamma} \iint\left(e^{\gamma t}\left(\int e^{-\gamma t} f_{t r}^{*} d t\right)-e^{-\gamma t}\left(\int e^{\gamma t} f_{t r}^{*} d t\right)+C_{1}^{*} e^{\gamma t}-C_{2}^{*} e^{-\gamma t}\right) d t^{2} .
$$

Influence upon (33), (27) of all inverse integral transformations $S_{i}^{-1},(i=\overline{1,3})$ that are responsible for originally applied, gives the wanted solutions of the general wave equation (GWE) (4) with respect to all scalar components of electromagnetic field vector intensities

$$
F=F(x, y, z, t)=\prod_{i=1}^{3} S_{i}^{-1} F_{t r} .
$$

In (34), expressions for $F_{t r}$ are defined by (27), (33).

Unexcited isotropic homogeneous linear medium.

Now, let the unexcited isotropic homogeneous medium be considered, and it differs from the preceding one by the presence of $\sigma>0$. Even such distinction as $\sigma \neq 0$, generates essential complication of GWE in comparison with (4),

$$
\partial_{0}\left(\partial_{0}+\frac{\sigma}{\varepsilon_{a}}\right)\left(\partial_{0}\left(\partial_{0}+\frac{\sigma}{\varepsilon_{a}}\right)-\frac{\Delta}{\mu_{a} \varepsilon_{a}}\right) F=\frac{f}{\left(\mu_{a} \varepsilon_{a}\right)^{2}},
$$

which the known function from the right part is written below

$$
f=\left(\partial_{i}^{2}-\mu_{a} \varepsilon_{a} \tilde{\partial}_{0}^{2}\right) \varphi_{k i}+\partial_{i}\left(\partial_{v} \varphi_{k v}+\partial_{l} \varphi_{k l}\right) ; v \neq l, i \neq v, i \neq l,(i, v, l=\overline{1,3} ; k=1,2) ;
$$




$$
\tilde{\partial}_{0}^{2}=\mu_{a} \varepsilon_{a} \partial_{0}\left(\partial_{0}+\frac{\sigma}{\varepsilon_{a}}\right)
$$

It is clear, that the difference between $f$ in (36) and the similar expression (5) consists only in appearance of operator $\tilde{\partial}_{0}^{2}$ instead of $\partial_{0}^{2}$, while functions $\vec{\varphi}_{k},(k=1,2)$ are completely identical to those from (5). Denoting differential operator

$$
\bar{\partial}_{0}^{2}=\partial_{0}\left(\partial_{0}+\frac{\sigma}{\varepsilon_{a}}\right)
$$

and the unknown function

$$
\bar{\partial}_{0}^{2} F=\Phi,
$$

one gets the simplified equivalent form of the original GWE

where $f$ is from (36);

$$
\left(\bar{\partial}_{0}^{2}-\frac{\Delta}{\mu_{a} \varepsilon_{a}}\right) \Phi=\frac{f}{\left(\mu_{a} \varepsilon_{a}\right)^{2}}
$$

$\Phi$ is described in (38);

$\bar{\partial}_{0}^{2}$ is given by (37).

As it is previous shown, all corresponding integral transformations that are applied to (39) by the spatial arguments $(x, y, z)$, lead to the ODE in terms of transforms regarding temporal variable $t$

$$
\left(\frac{d}{d t}\left(\frac{d}{d t}+\frac{\sigma}{\varepsilon_{a}}\right)-\frac{\Delta_{t r}}{\mu_{a} \varepsilon_{a}}\right) \Phi_{t r}=f_{t r}^{*}
$$

In (40) $\Delta_{t r}$ remains almost the same as in (11), accurate within change of $F$ to $\Phi$ everywhere in (6)...(9), (11), and

$$
f_{t r}^{*}=f_{t r}^{*}(t, p)=\frac{f_{t r}}{\left(\mu_{a} \varepsilon_{a}\right)^{2}}-\sum_{i=1}^{3}\left(\prod_{\substack{v=1 \\ v \neq i}}^{3} \int_{a_{v}}^{b_{v}} K_{v} d x_{v}\right) S_{i} .
$$

All other symbols in the round brackets from (41) are described previously concerning (11), and change of $F$ to $\Phi$ is mentioned above.

Solution of linear ODE with constant coefficients (40), (41) is done as previously, by the well known classical methods [5]. So the roots of the performance equation for (40) are

$$
\omega_{1,2}=-\frac{\sigma}{2 \varepsilon_{a}}\left(1 \mp \sqrt{1+\frac{4 \varepsilon_{a} \Delta_{t r}}{\mu_{a} \sigma^{2}}}\right)
$$

and completely depend on $\Delta_{t r}$ since $\sigma, \mu_{a}, \varepsilon_{a}=$ const $>0$ basing on the original conditions for GWE (1). Therefore as it is shown previously three versions of (42) should be considered:

1. $1+\frac{4 \varepsilon_{a} \Delta_{t r}}{\mu_{a} \sigma^{2}}>0 \Leftrightarrow \Delta_{t r}>-\frac{\mu_{a} \sigma^{2}}{4 \varepsilon_{a}} \in \mathbf{R}$, and $\omega_{1,2}$ are two different real roots from (42);

2. $1+\frac{4 \varepsilon_{a} \Delta_{t r}}{\mu_{a} \sigma^{2}}<0 \Leftrightarrow \Delta_{t r}<-\frac{\mu_{a} \sigma^{2}}{4 \varepsilon_{a}}<0, \quad \omega_{1,2}=-\frac{\sigma}{2 \varepsilon_{a}}\left(1 \mp i \sqrt{-\left(1+\frac{4 \varepsilon_{a} \Delta_{t r}}{\mu_{a} \sigma^{2}}\right)}\right), i=\sqrt{-1}$, expression under the symbol of $\sqrt{ }$ is positive, and $\omega_{1,2}$ is the pair of complex conjugate roots;

$$
\text { 3. } 1+\frac{4 \varepsilon_{a} \Delta_{t r}}{\mu_{a} \sigma^{2}} \in \mathbf{C} \Leftrightarrow \Delta_{t r} \in \mathbf{C}, \Delta_{t r}=\alpha+i \beta, \alpha=\operatorname{Re} \Delta_{t r}, \beta=\operatorname{Im} \Delta_{t r} ; \omega_{1,2}=-\frac{\sigma}{2 \varepsilon_{a}}\left(1 \mp\left(x^{*}+i y^{*}\right)\right),
$$


where $\sqrt{\left(1+\frac{4 \varepsilon_{a}}{\mu_{a} \sigma^{2}} \alpha\right)+\frac{4 \varepsilon_{a}}{\mu_{a} \sigma^{2}} \beta}=x^{*}+i y^{*}, \quad x^{*}= \pm \frac{1}{2}\left(1+\frac{4 \varepsilon_{a}}{\mu_{a} \sigma^{2}} \alpha+\sqrt{\left(1+\frac{4 \varepsilon_{a}}{\mu_{a} \sigma^{2}} \alpha\right)^{2}+4\left(\frac{2 \varepsilon_{a}}{\mu_{a} \sigma^{2}} \beta\right)^{2}}\right.$, $y^{*}=\frac{2 \varepsilon_{a} \beta}{\mu_{a} \sigma^{2} x^{*}}$.

Taking into account numerical values and structure of $\omega_{1,2}$ in all three cases from (43), FSS for the homogeneous equation respective to (40) can be written uniformly $\left\{\exp \left(\omega_{1} t\right), \exp \left(\omega_{2} t\right)\right\}$. Simultaneously, it forms the general solution of the inhomogeneous equation (40) for all cases (43)

$$
\Phi_{t r}=\Phi_{t r}(t, p)=C_{1}(t, p) \exp \left(\omega_{1} t\right)+C_{2}(t, p) \exp \left(\omega_{2} t\right) .
$$

Equation (44) is sought using method of arbitrary-constant variation [5]. In other words, the unknown functions $C_{j}(t)=C_{j}(t, p),(j=1,2)$ represent the solution of the following system

and are determined by formulae

$$
\left\{\begin{array}{l}
C_{1}^{\prime} \exp \left(\omega_{1} t\right)+C_{2}^{\prime} \exp \left(\omega_{2} t\right)=0 ; \\
C_{1}^{\prime} \omega_{1} \exp \left(\omega_{1} t\right)+C^{\prime} \omega_{2} \exp \left(\omega_{2} t\right)=f_{t r}^{*},
\end{array} C_{j}^{\prime}=\frac{\partial C_{j}}{\partial t},(j=1,2)\right.
$$

$$
C_{1,2}(t, p)= \pm \frac{1}{\omega_{1}-\omega_{2}} \int \exp \left(-\omega_{1,2} t\right) f_{t r}^{*}(t, p) d t+C_{1,2}^{*}(p)
$$

where $C_{1,2}^{*}(p)$ have the same meaning as in the previous paragraph.

Further substitution of (45) into (44) gives

$$
\begin{gathered}
\Phi_{t r}=\frac{1}{\omega_{1}-\omega_{2}} \times \\
\times\left(\exp \left(\omega_{1} t\right) \int \exp \left(-\omega_{1} t\right) f_{t r}^{*}(t, p) d t-\exp \left(\omega_{2} t\right) \int \exp \left(-\omega_{2} t\right) f_{t r}^{*}(t, p) d t+C_{1}^{*}(p) \exp \left(\omega_{1} t\right)-C_{2}^{*}(p) \exp \left(\omega_{2} t\right)\right) .
\end{gathered}
$$

In its turn, explicit expression (46) allows solving (38), (37) by means of the same transforms that is used for (39), (40). Namely,

$$
\frac{d}{d t}\left(\frac{d}{d t}+\frac{\sigma}{\varepsilon_{a}}\right) F_{t r}=\Phi_{t r} .
$$

It is clear, that (47) is the particular operator case of (40), but accurate within respective changes: instead of $\Phi_{t r}$ in (40), here $F_{t r}$ appears; $f_{t r}^{*}$ from (40) becomes $\Phi_{t r}$ from (46), and the latter is known already; at last, $\frac{\Delta_{t r}}{\mu_{a} \varepsilon_{a}}$ from the left part of (40) degenerates into zero for (47), though $\frac{\Delta_{t r}}{\mu_{a} \varepsilon_{a}}$ remains in the formula of $\Phi_{t r}$ during all preceding computations relating to (40).

ODE (47) is the second order linear inhomogeneous with constant coefficients, its characteristic equation has two roots $\left\{0,-\sigma / \varepsilon_{a}\right\}$, and corresponding FSS $\left\{1, \exp \left(-\left(\sigma / \varepsilon_{a}\right) t\right)\right\}$ forms the general solution of (47) [5]

$$
F_{t r}=F_{t r}(t, p)=C_{1}(t, p)+C_{2}(t, p) \exp \left(-\left(\sigma / \varepsilon_{a}\right) t\right) .
$$

As earlier, the unknown functions $C_{j}(t)=C_{j}(t, p),(j=1,2)$ in (48) are found by the method of arbitrary-constant variation from the system

$$
\left\{\begin{array}{l}
C_{1}^{\prime}+C_{2}^{\prime} \exp \left(-\left(\sigma / \varepsilon_{a}\right) t\right)=0 ; \\
C_{2}^{\prime}\left(-\sigma / \varepsilon_{a}\right) \exp \left(-\left(\sigma / \varepsilon_{a}\right) t\right)=\Phi_{t r},
\end{array} C_{j}^{\prime}=\frac{\partial C_{j}}{\partial t},(j=1,2)\right.
$$

and are given by formulae

$$
C_{1}(t, p)=\frac{\varepsilon_{a}}{\sigma} \int \Phi_{t r}(t, p) d t+\bar{C}_{1}^{*}(p), C_{2}(t, p)=-\frac{\varepsilon_{a}}{\sigma} \int \exp \left(\left(\sigma / \varepsilon_{a}\right) t\right) \Phi_{t r}(t, p) d t-\bar{C}_{2}^{*}(p),
$$

where $\bar{C}_{1,2}^{*}(p)$ have the same meaning as the previous constants $C_{1,2}^{*}(p)$. 
Substitution of (49) for (48) forms the required solution of (47). It represents the transform for all scalar components of electromagnetic field vector intensities

$$
\begin{gathered}
F_{t r}=F_{t r}(t, p)=\frac{\varepsilon_{a}}{\sigma}\left(\int \Phi_{t r}(t, p) d t-\exp \left(-\left(\sigma / \varepsilon_{a}\right) t\right) \int \exp \left(\left(\sigma / \varepsilon_{a}\right) t\right) \Phi_{t r}(t, p) d t\right)+ \\
+\bar{C}_{1}^{*}(p)-\exp \left(-\left(\sigma / \varepsilon_{a}\right) t\right) \bar{C}_{2}^{*}(p) .
\end{gathered}
$$

Analogy between (50) and (34) is obvious. Application to (50)

$$
F=F(x, y, z, t)=\prod_{i=1}^{3} S_{i}^{-1} F_{t r},
$$

creates unknown solutions of the GWE (35) describing all scalar components of electromagnetic field vector intensities in the framework of physical engineering problem statement. Formula, $S_{i}^{-1}(i=\overline{1,3})$, as earlier, determine all inverse integral transformations regarding originally used.

Conclusions. Both specific electrodynamic problems generated by the expofunctional influences and mathematically simulated by the general wave equations regarding all components of the electromagnetic field vector intensities, are solved explicitly in detail. Obtained final formulae are irrespective to the concrete boundary conditions and unite all required components of intensities. Rigorous results allow studying necessary engineering and physical effects using given analytic expressions with their appropriate direct computation and without any approximate calculus. Suggested approach can be applied to any kind of the finite dimensional system of PDEs with piece wise constant coefficients and its corresponding scalar equations representing mathematical models in current electrodynamics.

\section{Література}

1. Dmitrieva, I.Yu. Diagonalization of the differential operator matrix in the case of the multidimensional circuits / I.Yu. Dmitrieva, A.M. Ivanitckiy // Наукові праці ОНАЗ ім. О.С. Попова. — 2009. — № 1. C. $36-51$.

2. Dmitrieva, I. Some cases of electromagnetic wave propagation in terms of analytical study / I. Dmitrieva // Proceedings of the International Symposium on Signals, Circuits and Systems (ISSCS'2013), July 11-12, 2013, Iasi, Romania. — Piscataway, NJ: IEEE, 2013. - PP. 1 - 4.

3. Dmitrieva, I. On the solution of some differential equation in the classical Maxwell theory / I. Dmitrieva // Hyperion International Journal of Econophysics \& New Economy. - 2009. - Vol. 2, Issue 2. PP. $151-164$.

4. Tranter, C.J. Integral Transforms in Mathematical Physics / C.J. Tranter. $-2^{\text {nd }}$ Ed. - London: Methuen; New York: Wiley, 1956. - 133 p.

5. Камке, Э. Справочник по обыкновенным дифференциальным уравнениям / Э. Камке; пер. С.В. Фомин. - 5-е изд., стер. - М.: Наука, 1976. - 576 с.

\section{References}

1. Dmitrieva, I.Yu. and Ivanitckiy, A.M. (2009). Diagonalization of the differential operator matrix in the case of the multidimensional circuits. Proceedings of the O.S. Popov ONAT, 1, 36-51.

2. Dmitrieva, I. (2013). Some cases of electromagnetic wave propagation in terms of analytical study. In Proceedings of the International Symposium on Signals, Circuits and Systems (ISSCS'2013) (pp. 1-4). Piscataway, NJ: IEEE.

3. Dmitrieva, I. (2009). On the solution of some differential equation in the classical Maxwell theory. $H y$ perion International Journal of Econophysics \& New Economy, 2(2), 151-164.

4. Tranter, C.J. (1956). Integral Transforms in Mathematical Physics ( $2^{\text {nd }}$ Ed.). London: Methuen; New York: Wiley.

5. Kamke, E. (1965-1967). Differentialgleichungen. Lösungsmethoden und Lösungen (Vols. 1-2). Akademische Verlagsgesellschaft. 